\section{REVISTA}

Actualidades Investigativas en Educación http://revista.inie.ucr.ac.cr/

ISSN 1409-4703

\title{
EXPERIENCIA DE LA DEFINICIÓN DE COMPETENCIAS EN LA ESCUELA DE ADMINISTRACIÓN PÚBLICA
}

SOME EXPERIENCES ABOUT COMPETENCY MODEL IN PUBLIC ADMINISTRATION $\mathrm{SCHOOL}$

\section{Volumen 15, Número 3}

Setiembre - Diciembre

pp.1-31

Este número se publicó el $1^{\circ}$ de setiembre de 2015

DOI: http://dx.doi.org/10.15517/aie.v15i3.20981

Angélica Vega Hernández

Revista indizada en REDALYC, SCIELO

Revista distribuida en las bases de datos:

CATÁLOGO DE LATINDEX, IRESIE, CLASE, DIALNET, DOAJ, E-REVIST@S, SHERPA/ROMEO, QUALIS, MIAR

Revista registrada en los directorios:

ULRICH'S, REDIE, RINACE, OEI, MAESTROTECA, PREAL, CLACSO 


\title{
EXPERIENCIA DE LA DEFINICIÓN DE COMPETENCIAS EN LA ESCUELA DE ADMINISTRACIÓN PÚBLICA \\ SOME EXPERIENCES ABOUT COMPETENCY MODEL IN PUBLIC ADMINISTRATION SCHOOL
}

\begin{abstract}
Angélica Vega Hernández ${ }^{1}$
Resumen: Se presenta una sistematización del proceso llevado a cabo en la Escuela de Administración Pública de la Universidad de Costa Rica para desarrollar un curriculum académico por competencias. El proceso consistió en un esfuerzo de la organización con un fuerte componente de trabajo en equipo, que permitiera el aprendizaje de la organización en el tema de competencias profesionales, la valoración del objeto de estudio de la administración pública y consecuentemente diseñar el currículum por competencias para el profesional de la administración pública. El esfuerzo generó la definición de competencias generales y específicas, y la organización curricular en módulos, de tal forma que se alcanzó una estructura de plan de estudios totalmente diferente a lo que se ha venido construyendo en la Universidad de Costa Rica.

Palabras clave: GESTIÓN POR COMPETENCIAS, EDUCACIÓN SUPERIOR, GESTIÓN DEL CAMBIO, MALLA CURRICULAR, COMPETENCIAS, UNIVERSIDAD DE COSTA RICA.

Abstract: The following presents the process executed by Public Administration School that allowed a new curriculum in competencies. The process required excellent team work, where professors were tasked with learning about competencies, reviewing public administration theory and finally building a professional competencies module. The effort allowed the definition of specific and general competencies and design modules instead of courses. The Public Administration project is a new propoal been built and implemented for the first time at the University of Costa Rica. The article concludes that it is possible to implement modernization processes in the curriculum that break current paradigms. Also provides recommendations on processes of joint construction that impact positively on the review of discipline and improving processes teaching and learning.
\end{abstract}

Keywords: COMPETENCY MODEL, HIGHER EDUCATION, CHANGE MANAGEMENT, CURRICULUM, COMPETENCIES, UNIVERSITY OF COSTA RICA.

\footnotetext{
${ }^{1}$ Docente en Régimen de la Universidad de Costa Rica y coordinadora del proceso de cambio al modelo por competencias de la carrera de Administración Pública.
}

Dirección electrónica: angelica.vega@ucr.ac.cr

Artículo recibido: 12 de diciembre, 2013

Enviado a corrección: 25 de febrero, 2014

Aprobado: 17 de agosto, 2015 
“¿Me podrías indicar hacia donde tengo que ir desde aquí?” pregunta Alicia. "Eso depende de a dónde quieras llegar" responde el gato. "A mi no me importa demasiado a donde"." En ese caso, da igual hacia donde vayas"-"Siempre que llegue a alguna parte""iOh! Siempre llegarás a alguna parte, si caminas lo bastante".

El Gato y Alicia en el país de las maravillas

\section{Introducción}

Cuando de competencias se trata, hay un vasto campo de conocimiento profesional generado en el ámbito internacional. La Organización Internacional del Trabajo (OIT) desarrolla grandes esfuerzos por consolidar procesos de formación, desarrollo y certificación por competencias. Sin embargo, el camino de las competencias es algo propio, posiblemente la metodología general pueda replicarse pero el proceso de reflexión es singular, permanente y continuo, su final siempre es revalorado.

La frase del Señor Gato de Alicia en el país de las maravillas comúnmente es utilizada para forzar a las personas a definir un lugar de llegada. En las páginas de este documento la pregunta de Alicia y la del Gato tendrán sentido en la medida en que el punto de llegada es importante, pero el rumbo, el pasaje o la vivencia que, reconociendo era necesario para que Alicia comprendiera la razón de su presencia, es igualmente relevante. El camino que toma Alicia le permite encuentros con diversos personajes y las decisiones que toma son lo que finalmente quedan como parte de las enseñanzas.

En esta historia el final no constituye lo importante para Alicia -aunque era lo que deseaba- sino más bien lo valioso fue su viaje, su aprendizaje y sus vivencias. Esa misma es la riqueza de un plan por competencias.

\section{¿Hacia dónde queremos llegar con esta metáfora?}

Cuando se busca un cambio en el enfoque por competencias en los planes curriculares se verá cómo poco a poco se adentra en un mundo lleno de preguntas sin respuestas, y se espera que el rumbo tenga la capacidad de redefinir el puerto de llegada.

Siguiendo la recomendación del Gato de Alicia llegaremos a alguna parte si caminamos lo bastante, ya que un proceso de reflexión es un andar permanente y ese camino, precisamente, redefine nueva...s formas y rumbos. El proceso que nos forma y nos lleva al conocimiento así como la utilidad de este conocimiento para el ser humano es lo importante, no el conocimiento como tal. 
Este documento sistematiza una experiencia que se desarrolla desde el año 2007. La decisión de compartirlo se ha tomado por aquellas personas que buscan algunas respuestas a sus preguntas y percepciones respecto a las competencias. Este documento pretende apoyarle como agente de cambio al conocer una experiencia que le puede permitir tomar decisiones y prever situaciones sobre cómo discutir el tema de competencias en su unidad académica y definirlas para su perfil profesional. Se presenta la metodología seguida, junto con la experiencia de cómo fue abordado el proceso.

\section{1. ¿Por qué cambiar el currículo hacia competencias?}

En el campo de la administración pública varios países como Alemania, Francia, Canadá e Inglaterra han implementado el enfoque de capacidades y competencias como parte de sus procesos de contratación y formación profesional. La consolidación profesional debe proponer la reflexión sobre la relevancia de la disciplina y la labor profesional, amalgamando capacidades que, algunas veces, están fuera de nuestro ámbito educativo pero que, sin embargo, son relevantes en la construcción de un perfil profesional competente. Esto no es un fin en sí mismo, lo importante es descubrir cómo nuestra disciplina realmente es valiosa para la sociedad.

Cuando se ingresa como docente a la vida académica universitaria, es normal que la persona se sienta afortunada de tener la oportunidad de formar a futuros profesionales. Posterior a la sensación de sentirse afortunado, procede una leve preocupación sobre qué temas son más importantes para transmitir a los estudiantes. Pensamos entonces en nuestro mejor profesor o profesora y tratamos de recordar todo aquello que nos llamaba la atención y nos inspiraba respeto. Igualmente, a los profesores menos afortunados que no gozan de tal estima y pensamos en cómo no ser parecidos. En fin, retomamos los temas de los programas de ciclos anteriores y tratamos de mejorarlos, actualizarlos y de incluir un par de amigos que pueden dar las charlas y una película que puede resultar de ayuda.

A pesar del júbilo con que comenzamos nos damos cuenta, luego de varios ciclos de dictar el curso, que hay estudiantes que no comprenden nuestro mensaje, que no les agradamos, o que simplemente creemos que no les interesa. Tenemos en alta estima a nuestros alumnos "promedio" que se acercan a nuestro estereotipo de "buen estudiante" a los cuales les auguramos una excelente vida profesional. Sin embargo, cuando trabajan nos damos cuenta de que los buenos no eran tan buenos y los malos, en realidad, no lo eran tanto, puesto que ocupan cargos gerenciales o de dirección, hacen estudios de posgrado a 
pesar de que en nuestro curso nunca los hubiéramos considerado para ningún reconocimiento (Livinstong, 1971).

Lo anterior, resume el proceso normal del diseño educativo tradicional con preconcepciones marcadas como:

a. Los estudiantes necesitan conocimientos para prepararse como profesionales, para eso están en las aulas.

b. El y la estudiante buenos o excelentes son aquellos que responden de manera acertada a todos los conocimientos y recomendaciones que he dado en clases.

c. Los estudiantes van a aprender realmente cuando trabajen, por ahora solo necesitan las bases teóricas.

d. Un estudiante que es exitoso o exitosa en la universidad, de manera inequívoca va a ser un profesional exitoso.

Tomando como base lo anterior, el docente tomará las siguientes decisiones y posiciones respecto al proceso educativo:

- Mi aporte al conocimiento de los estudiantes es asegurarme de que dominen todo lo que puedan de mi materia para que lo apliquen en sus trabajos.

- Cuantos más contenidos vea y más aproveche el tiempo, mejor profesor o profesora soy.

- Los malos estudiantes (no cumplen con el estereotipo) deben ser personas que no tienen objetivos en la vida, que desaprovechan la oportunidad universitaria.

- Un indicador de lo buen o mal profesor o profesora es cuántas personas consideran difícil y pierden mi curso, por eso debe haber siempre un porcentaje de reprobados. Además los estudiantes esperan de mi perdedores y ganadores, cuanto más difícil sea el curso representará un mayor desafío para ellos.

- Los mejores indicadores son las calificaciones de exámenes y trabajos finales. Así lo hice yo y no me considero una persona traumatizada por ello.

Aunque todo lo anterior no implica, de ninguna manera, que los profesores y profesoras no se esfuercen, es muy probable que sus resultados sean parcialmente buenos, ya que el objetivo del curso no se cumple a cabalidad y las capacidades no se garantizan en los y las jóvenes en su totalidad. El asunto medular de esta reflexión es que no estamos 
hablando solo de estudiantes universitarios y universitarias, nos referimos a futuros profesionales.

El iniciar el camino hacia las competencias implica reconocer qué queremos para nuestra disciplina, acaso son profesionales con enfoques técnicos y especializados en materias concretas, o bien perfiles menos especializados y más integrales con capacidades relevantes que les permitan desenvolverse adecuadamente en una buena parte de su vida profesional. Esto implica enfocarse en tres preguntas:

- ¿Cómo queremos que el profesional sea y se desenvuelva?

- ¿Qué queremos que haga en su trabajo y bajo cuáles estándares de calidad?

- ¿Cómo podemos ayudarle a evolucionar y a aprender a lo largo de su vida?

El por qué cambiar hacia las competencias es una convicción de la comunidad académica e implica una responsabilidad con los estudiantes. En el caso de la administración pública, esto constituye un desafío al ser un campo de acción tan amplio y diverso pero, a la vez, relevante para la sociedad.

\subsection{La tendencia internacional de sistemas de certificación de la calidad educativa y profesional: ¿Preparamos para procesos de mejoramiento continuo?}

En Costa Rica el Sistema de Acreditación de la Educación Superior (SINAES) constituye un ente adscrito al Consejo Nacional de Rectores (CONARE) y tiene como misión certificar procesos de mejoramiento de la calidad en los sistemas educativos de las carreras sometidas a dicho análisis.

La realización de estos procesos de certificación en las carreras universitarias son poco frecuentes, por lo que en la Universidad de Costa Rica al momento de iniciar este cambio de enfoque curricular, existían solamente nueve carreras acreditadas por SINAES, dos de las cuales pertenecen a la Escuela de Administración Pública. El proceso de mejoramiento de la calidad llevó a la Escuela a plantearse y replantearse la siguiente pregunta ¿Qué incidencia tiene la carrera de administración pública en la sociedad costarricense y en el ámbito internacional?

En este nuevo enfoque de la calidad, basado en competencias, los indicadores cuantitativos pasan a un segundo plano para dar cabida a preguntas como: 
- ¿Cuántos profesionales graduados han implementado o implementan procesos de cambio y decisión en la función pública?.

- ¿Cuántas publicaciones de la Escuela constituyen herramientas de gestión en la realidad institucional costarricense?.

- ¿Cómo las nuevas tecnologías impactan positivamente el proceso educativo de la Escuela y son incorporadas de manera natural en la toma de decisiones?.

- ¿Qué competencias reales pueden, los estudiantes, indicar que poseen, que les permitan desenvolverse en el ámbito nacional e internacional?

En muchas de las carreras con un enfoque tradicional, estas preguntas tienen poca 0 ninguna relevancia cuando no existe un verdadero enfoque hacia los resultados. El proceso de certificación de la calidad del SINAES constituyó un detonante de la necesidad de replantear un proceso educativo que, en primer lugar, pasara de pedagógico a uno andragógico $^{2}$, de reconocer que los y las estudiantes llegan a la universidad con capacidades y realidades diferentes y eso implica procesos de aprendizaje diversos.

Otro aspecto que impulsó el proceso de mutabilidad fue el cambio generacional, donde, precisamente, los anteriores estudiantes que realizaron sus estudios de posgrado regresaron a la Escuela como profesores, con nuevas y mejores propuestas a las vigentes en el momento.

La gestión curricular constituye una serie de decisiones relacionadas con procesos de mejora continua y calidad en las carreras, por lo que de ninguna manera constituye un fin en sí mismo. Uno de los propósitos de la metodología por competencias es formar profesionales que consideren valiosos los procesos de mejora continua y replanteamiento del status quo, situación de gran relevancia en la administración pública. Se considera que solo a través de un proceso vivencial los futuros profesionales pueden adquirir capacidades, valores y destrezas que les permitan generar un cambio en lo público con incidencia a nivel nacional e internacional.

\footnotetext{
${ }^{2}$ Las clases en el ámbito universitario obvian la necesidad de comprender que existe una diversidad de personas con necesidades diferentes. Por ejemplo tenemos a los adolescentes que llegaron muy rápidamente al sistema educativo de forma exitosa e ingresan a la universidad de 16 o 17 años, los adultos jóvenes quienes por diversas razones están en la Universidad a los 20 o 25 años. En años recientes hay una mayor cantidad de adultos y adultos mayores. Cuando un grupo resume todas estas poblaciones se debe tener cuidado con el enfoque pedagógico/ andragógico que se debe asumir, porque además las formas de aprendizaje son diversas entre adolescentes, adultos y adultos mayores. El proceso por competencias nos lleva irremediablemente a determinar estas necesidades en cada uno de los estudiantes.
} 


\subsection{La interconexión profesional y académica en un ambiente globalizado.}

Cuando este tipo de procesos de cambio se inician es lógico considerar las preguntas ¿Qué es y qué implica desarrollar nuestra disciplina? Y ¿cuál es el papel desempeñado por esta en la sociedad?, y más concretamente ¿Qué papel cumple una escuela de administración pública en la sociedad? Cuando un conjunto de personas inician un proceso de reconocimiento profesional y disciplinario de este tipo, las preguntas llevan a un debate teórico y práctico respecto a las posibles capacidades profesionales más relevantes y proyectando los objetivos compartidos entre la academia y la sociedad que las posibiliten.

En su libro "Diseño, desarrollo e innovación del currículo", Escudero (2009) comenta sobre las raíces de la complejidad del cambio educativo y brinda una recomendación amplia e importante relacionada con el respeto que se debe guardar en el proceso de análisis de la complejidad disciplinaria hacia un cambio curricular y cita:

Muchas políticas de reforma suelen actuar todavía como si de un asunto fácil y expedito se tratara: dan por buenas sus propuestas y, sólo por remitirlas a los centros y profesores las consideran realizables. De forma parecida ciertas apreciaciones y desafecciones sociales, profesionales y personales con las mismas surgen, además de los sobrados motivos externos para ello, de una falsa imagen respecto a lo que son y comportan los cambios en educación, así como de expectativas infundadas en su poder para solucionar los problemas vividos y sentidos. El malestar con las reformas puede incrementarse todavía más si renunciamos de ir mas allá de los tópicos al uso, si son tratadas con desidia y falta de respeto, o si entendemos que, ya que prometen mejoras, solo por el hecho de que se diseñen y apliquen han de desaparecer las dificultades. (Escudero, 2009, p. 79)

El cambio curricular por competencias ofrece altas posibilidades de mejora que, solamente, dependen de la capacidad que tenga la comunidad docente para interiorizar el cambio, así como de lo que representa su disciplina y la relevancia o papel social de sus profesionales. Un cambio por competencias que no contemple los elementos establecidos en el Diagrama 1, sin acciones reflexivas, que se piense como fin de innovación de modelo y no como proceso, provocará cambios temporales de corto alcance que, incluso, pueden llevar a mayores frustraciones por docentes y estudiantes. Nótese que en todo momento se habla de grupo y comunidad, esto implica que el trabajo por competencias no debe ser impulsado por 
una sola persona. Para que tenga sentido, debe ser una visión conjunta que permita acciones colaborativas.

\section{Diagrama 1}

\section{Entorno educativo como determinantes del currículo}

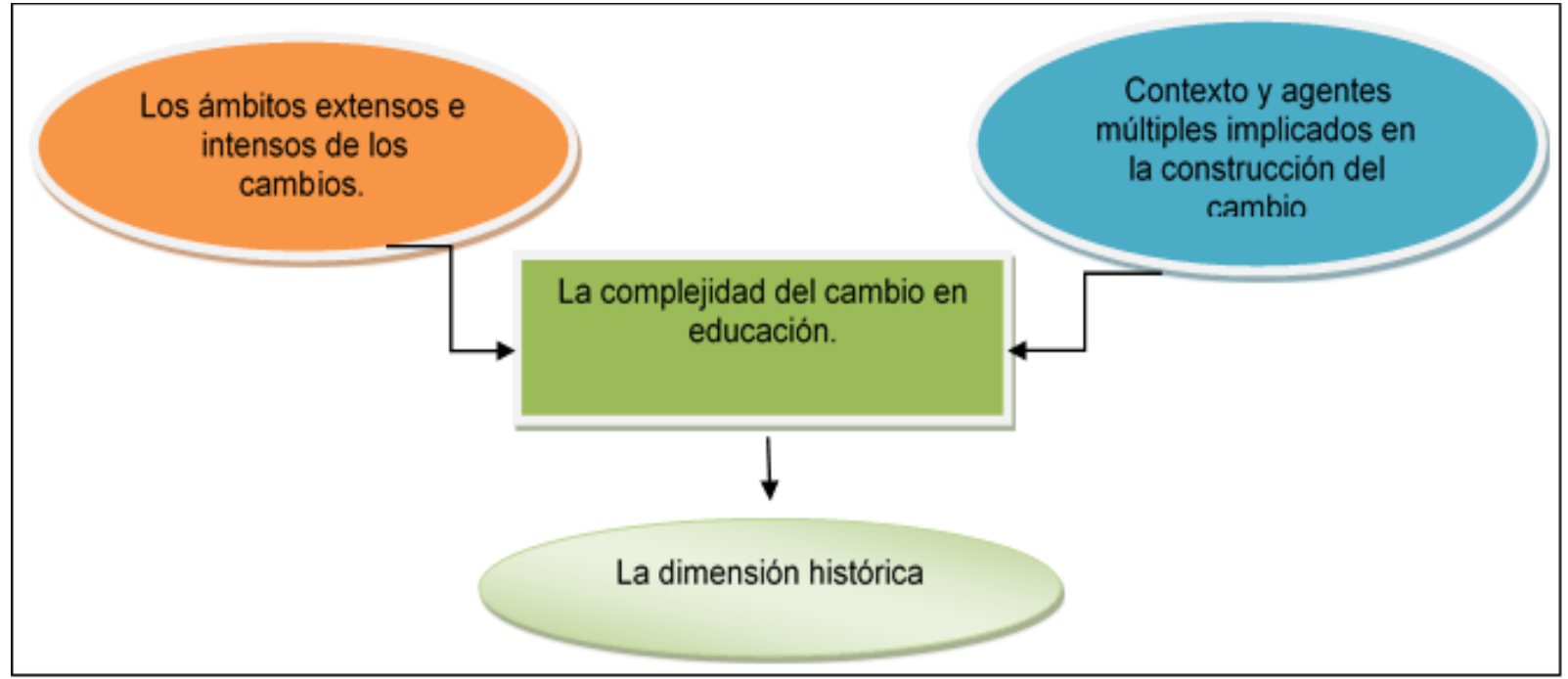

Fuente: Escudero (2009). Las raíces de la complejidad del cambio educativo.

El autor recomienda que se considere el ambiente (actual y futuro) del cambio educativo pretendido, para lo cual el enfoque histórico es fundamental. Es por esta razón que a inicios del año 2007 se consolidó la Comisión de Reforma Curricular en la Escuela con el fin de realizar las mejoras que fueron evidenciadas durante el proceso de CONARE y su primera labor fue definir el papel estratégico de la EAP en cuanto formadora y como parte de la gestión del conocimiento disciplinario.

La Escuela de Administración pública definió que su papel disciplinario se basa en:

1. Comprender al Estado como una relación social e histórica, donde la sociedad civil es el principal eje.

2. Tomar en cuenta la construcción social de la ciudadanía, y en ellos el fortalecimiento del análisis de lo público y de los procesos democráticos.

3. Considera a la organización social y humana como la base para el alcance de logros públicamente valorados.

4. Concebir a la administración pública como una manifestación en espacio y tiempo del Estado. 
5. Contribuir con la generación y aplicación de conocimiento relevante para la sociedad, por medio de la investigación disciplinaria, aplicada y sustentada en la transdisciplinariedad.

6. Permitir mecanismos de colaboración e investigación entre espacios académicos y profesionales, públicos y privados, que promuevan altos grados de gobernabilidad.

7. Promover el fortalecimiento de la gobernanza institucional y de las organizaciones sociales, a través de relaciones colaborativa y corresponsables entre la sociedad civil.

Para cada uno de los elementos disciplinarios supra citados, se desagregan a continuación las características profesionales (habilidades, conocimientos y comportamientos) que se desarrollarán en los estudiantes para ser aplicadas en la vida laboral: $\left({ }^{3}\right)$

1. Comprensión y análisis holístico de la sociedad: el profesional en administración pública integra los aportes de diferentes disciplinas para comprender y analizar los intercambios o acuerdos sociales, así como la evolución de las necesidades a las que el Estado debe responder.

2. Capacidad de análisis transdisciplinario: tiene capacidad comprender del contexto y el entorno diverso, sistematización de "realidades valoradas" por los actores, y ordenamiento de las causas y consecuencias de las necesidades en el ámbito nacional, interméstico, regional y local.

3. Diseño de metodologías y herramientas: conoce y es capaz de desarrollar nuevas metodologías para la determinación de requerimientos organizativos como recursos, aprovechamiento del talento humano, organización del trabajo, producción de servicios, alcance de objetivos, seguimiento y evaluación desde las organizaciones en que trabaja.

4. Comprensión del ordenamiento jurídico en la toma de decisiones: contempla el espacio de acción de la administración del Estado, y los mecanismos de participación de la sociedad civil en la esfera pública (derecho público y civil).

\footnotetext{
${ }^{3}$ Las clases en el ámbito universitario obvian la necesidad de comprender que existe una diversidad de personas con necesidades diferentes. Por ejemplo tenemos a los adolescentes que llegaron muy rápidamente al sistema educativo de forma exitosa e ingresan a la universidad de 16017 años, los adultos jóvenes quienes por diversas razones están en la Universidad a los 20 o 25 años. En años recientes hay una mayor cantidad de adultos y adultos mayores. Cuando un grupo resume todas estas poblaciones se debe tener cuidado con el enfoque pedagógico/ andragógico que se debe asumir, porque además las formas de aprendizaje son diversas entre adolescentes, adultos y adultos mayores. El proceso por competencias nos lleva irremediablemente a determinar estas necesidades en cada uno de los estudiantes.
} 
5. Desarrollo permanente de competencias y construcción del conocimiento: el profesional cuenta con la capacidad de potenciar sus competencias de liderazgo, negociación, comunicación, investigación y transferencia de conocimiento desde su espacio de acción que brinden valor agregado a su trabajo y sea extensivo a los demás miembros.

Lo anterior, parte de la premisa de que el y la profesional se enfrentan a problemas y necesidades globales y planetarias que requieren abordajes y respuestas sistémicas y de largo alcance. (Morín , 2009)

\section{2. ¿Cómo iniciamos un plan curricular por competencias?}

\section{1 ¿Incursionamos en las competencias?}

El desarrollo de un plan curricular por competencias no es un fin en sí mismo, es un instrumento de mejora. Algunas veces las universidades 0 escuelas se encuentran confundidas y presionadas por asumir el modelo de gestión por competencias, lo cual ha provocado que erróneamente se considere como una moda. Esta visión de cambio tan superficial más que apoyar los procesos de innovación educativa, puede terminar afectándolo.

Las apreciaciones y experiencias negativas, relacionadas con el desarrollo de planes curriculares por competencias, pueden deberse a que se visualizan desde cualquiera de los siguientes escenarios:

1. Considerar que la formación por competencias es una forma exótica y llamativa de educación en el ámbito internacional, por lo que se implementa de forma brusca en procesos educativos sin convicción real de un cambio, preparación y razón alguna más que por presión externa.

2. Tener la idea de que las competencias van a sustituir la preparación formal basada en contenidos, donde estos literalmente desaparecen. Nada más alejado de la verdad puesto que contenidos y capacidades profesionales se deben amalgamar en el currículo de la carrera.

3. Esperar que con el simple cambio de nombre de cursos a módulos y el diseño de algunas competencias (cuando no se toman literalmente de otra experiencia), generará un cambio espontáneo que, al no suceder, genera frustraciones. 
4. Creer que todos los problemas de la unidad van a solucionarse con el cambio hacia competencias. Se debe recordar que la gestión educativa lo es desde el espacio estratégico, administrativo, académico y de extensión social. El modelo curricular incide en lo académico pero debe haber un esfuerzo de integración hacia las otras áreas. En competencias no existe la generación espontánea, es un proceso de generación de consciencia y de acciones sostenidas.

\section{2 ¿Cuál es el punto de partida?}

Con base en todo lo anterior, se llega a la conclusión de que un cambio en las competencias es una DECISIÓN que el conjunto de docentes impulsores toman, considerando que la disciplina, el perfil profesional y la incidencia en la comunidad nacional e internacional se verán beneficiados con este cambio. También es necesario aclarar que las competencias no son la panacea que solucionará todos los problemas y desequilibrios en la gestión educativa, es un enfoque que permite una educación más integral.

Entonces el punto de partida será la convicción de que se necesita un cambio, las razones por las cuales se requiere y el compromiso de trabajo de un equipo de docentes, profesional administrativo y estudiantes. Cabe aclarar que aunque el proceso es en equipo, la persona que asuma la dirección del proceso debe contar con el apoyo de las autoridades institucionales. En el caso de la Escuela de Administración Pública esta pregunta se respondió de la manera descrita a continuación: Durante el período 2000-2002 la EAP desarrolló un proceso de acreditación nacional educativa donde el SINAES realizó una serie de recomendaciones a la carrera. Posterior a la acreditación de SINAES, la Escuela realizó una serie de compromisos de mejora, dentro de los cuales se encontraba el cambio de plan curricular.

La Comisión de Reforma Curricular se dio a la tarea de hacer un análisis sistémico de las necesidades de la Unidad Académica que estaban incidiendo negativamente en el y la profesional. El Diagrama 2 muestra los desafíos más importantes basados en un problema concertado, que resulta el más práctico y preocupante en la formación de la administración pública: "La capacidad limitada de la oferta educativa en la Escuela, para apoyar al estudiante en la determinación de un espacio de acción definido en la sociedad, y por consiguiente de generar un verdadero impacto en las instituciones a nivel nacional".

Para realizar el análisis se utilizó la herramienta de árbol de causas y consecuencias, basado en la Metodología de marco lógico. (Ortegón, Pacheco y Prieto, 2005). La Comisión 
consideró importante resaltar que, para lograr un cambio positivo que posicione a la Escuela como un actor relevante en la formación, actualización y evaluación de los profesionales en la función pública, no solo es necesario un cambio en el Plan de Estudios, sino que se requieren acciones sistémicas. Para realizarlo se desarrollaron talleres diversos con personas externas a la Comisión (profesores, estudiantes, egresados) y a la Escuela (empleadores, investigadores).

La discusión promovió un reforzamiento del capital social y académico necesario que permite posteriormente el proceso de implementación. $\left({ }^{4}\right)$ Actualmente, esta capacidad desarrollada permite llegar a acuerdos de forma mucho más rápida y expedita, reconociendo nuestras capacidades individuales y de grupo.

Construir un compromiso de este tipo es el punto de partida que, como en el pasaje, Alicia no se construye de forma ex ante, sino que se logra posteriormente a una decisión compartida de iniciar un trabajo conjunto. Es decir, primero debemos crear una Escuela y un espacio de trabajo adecuado y luego ofrecer a los estudiantes un espacio adecuado para su educación. Esta es una bonita oportunidad de lograrlo.

\footnotetext{
${ }^{4}$ Parte del compromiso que se debe resaltar se hace evidente al indicar que durante los años 2007, 2008 y 2009 la Comisión se reunía todos los días lunes en la Escuela, al finalizar los períodos laborales de cada miembro (la hora de inicio era a las 5:30 ó 6:00 pm), las sesiones de discusión variaban cada lunes entre tres y cinco horas.
} 


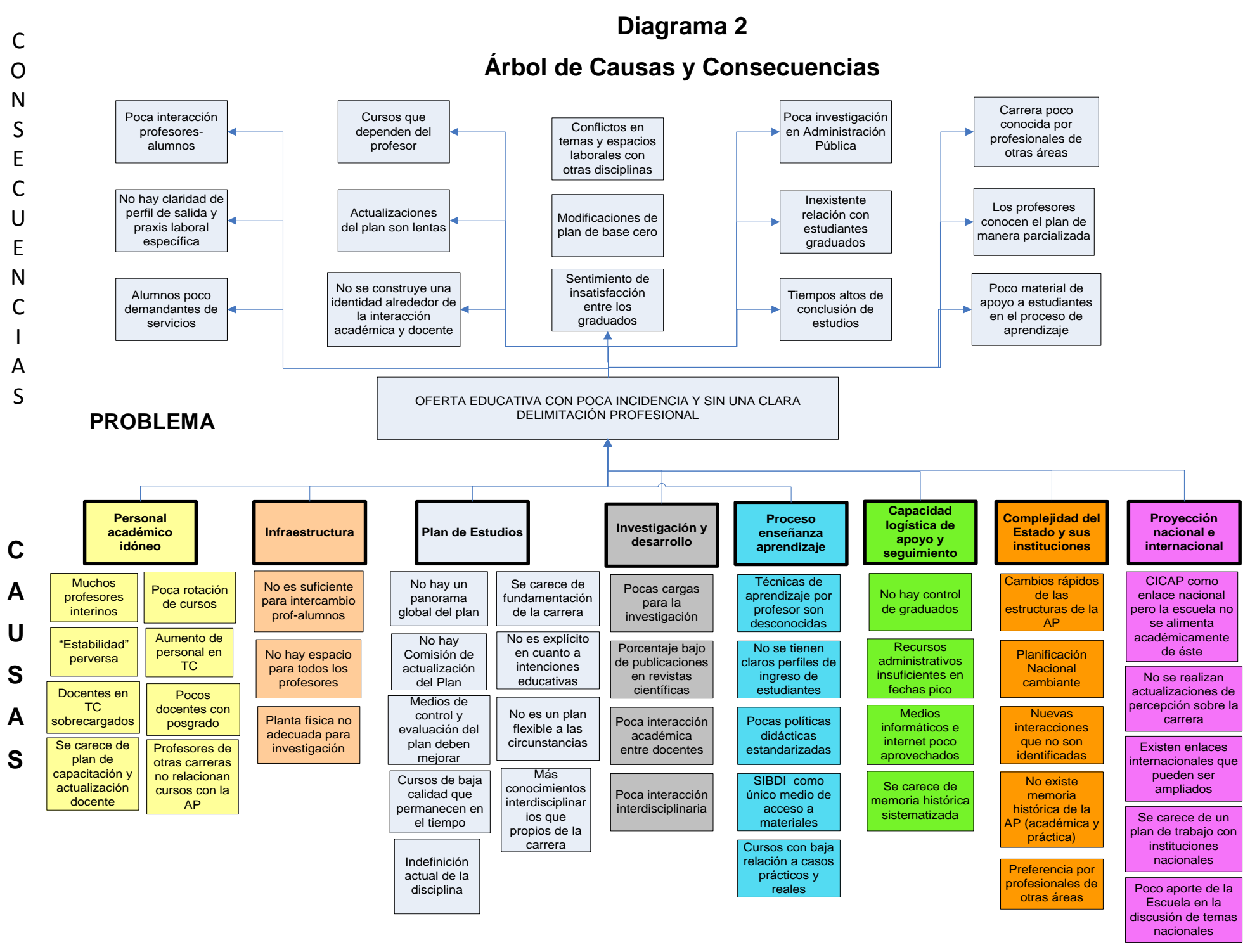

Fuente: Elaboración propia. 


\section{3 ¿Cómo construimos nuestro diagnóstico?}

Con la finalidad de brindar una mayor comprensión del Diagrama 2, a continuación, se explican brevemente las principales causas del problema, las cuales se identifican desde los siguientes elementos:

1. Personal académico: El análisis conlleva a considerar decisiones importantes como el aumento de plazas docentes en tiempo completo para profesores jóvenes que hicieran estudios de posgrado internacional, la generación de un plan de carrera para nuevos cuadros docentes, incremento de plazas para investigación y consolidación de enlaces nacionales e internacionales que permitan la actualización en temas, herramientas y metodologías.

2. Infraestructura: uno de las carencias más importantes es la falta de espacio para el intercambio docente, estudiantil y el desarrollo de la investigación. Se considera importante el diseño de espacios de intercambio para reuniones individuales o una con un grupo mayor.

3. Plan de estudios: revisión del objeto de estudio de la disciplina, análisis del perfil de salida, estandarización de contenidos de los cursos e interrelación entre las materias, fortalecimiento de capacidades docentes, así como la integración de contenidos o conocimientos hacia destrezas y capacidades profesionales de salida. ${ }^{5} \mathrm{El}$ estudio de la reforma curricular de 1990 mostró un exceso de requisitos y correquisitos, por lo que para el plan del año 2000 se decretó eliminar requisitos. Sin embargo durante la implementación del plan 2000-2010 fue evidente que la interconexión de capacidades que debían tener los estudiantes para ciertos cursos no se cumplía por la falta de un análisis previo de construcción de capacidades. Adicionalmente, del $100 \%$ de las materias del plan 2000 solamente $27 \%$ estaban relacionadas directamente con la disciplina, las demás materias eran cursos de servicio (economía, matemática) o cursos generales (humanidades).

4. Investigación y desarrollo: Se consideró importante la conformación de equipos de investigadores, su capacitación y apoyo para aumentar la cantidad de publicaciones.

5. Proceso de enseñanza-aprendizaje: Una de las principales preocupaciones era la forma como se induce a los nuevos docentes, por lo que un aspecto a resaltar fue la

\footnotetext{
${ }^{5}$ Cuando se indica capacidad docente, se refiere a los conocimientos, habilidades y destrezas del profesor que le permiten comprender el proceso de enseñanza-aprendizaje, determinar los objetivos didácticos de su curso, reconocer el perfil de entrada y salida del estudiante, y diseñar una estructura curricular sistemática. La docencia es una capacidad adicional cuyas bases y competencias deben ser desarrolladas.
} 
necesidad de procesos de formación para docencia en los profesionales de la Escuela. Es importante que se establezcan perfiles claros, así como también que se cuente con un proceso de inducción a nuevos profesores con el fin de entregar productos educativos sólidos a la comunidad estudiantil.

6. Capacidad administrativa, apoyo y seguimiento: La capacidad administrativa se consideró aceptable, sin embargo los nuevos cambios que se derivan de la implementación de un plan por competencias anuncian una preparación y modernización en este sentido.

7. Complejidad constante del Estado y sus instituciones: se reconoce la necesidad de construir enlaces de colaboración permanentes con actores nacionales que alimenten las actualizaciones curriculares. En esta misma línea es importante el seguimiento que se le brinde al administrador público dentro de las carreras administrativas de las instituciones, de tal forma que se consoliden espacios profesionales dentro de la función pública para nuestros profesionales.

8. Proyección nacional e internacional: ante esta coyuntura, la Escuela debe preguntarse ¿Queremos ser una escuela localista y que responda al espacio nacional o incursionar en el campo internacional? Entre los aspectos relevantes se encuentra la revisión de los enlaces y la dedicación de capital humano para reactivarlo.

Los elementos citados previamente demuestran que si bien un proceso de cambio curricular es relevante para un centro educativo, es importante también realizar un ejercicio paralelo que considere otros aspectos estructurales que van más allá de la oferta curricular. En este sentido, la EAP inicia un proceso de mejoramiento continuo con base en el apoyo y asesoría del SINAES.

\section{4 ¿Cómo llegamos al objeto de estudio?}

Antes de proceder al diseño de un plan curricular se deben llegar a dos acuerdos que respondan a las siguientes preguntas:

a. ¿Cuál es el perfil profesional que queremos desarrollar?

b. ¿Cuál es el objeto de estudio disciplinario que nuestra Escuela ha definido?

El responder a estas dos preguntas no resultará tarea sencilla, puesto que implica un acuerdo de la comunidad académica donde los profesores y estudiantes serían los primeros 
actores con la asesoría de los graduados y empleadores. Se presentan algunas acciones consideradas erróneas al definir este proceso:

1. Cuando un grupo pequeño de profesores responde a las preguntas profesionales y las define como lineamientos de toda la escuela. Esta forma jerárquica de decisiones, a la larga, provocará que nadie se comprometa con el proceso. Debemos tener un equipo de trabajo que funcione como motor de cambio pero también espacios de integración de la comunidad docente. ${ }^{6}$

2. Solicitar a un experto o experta para que defina el producto final. Es adecuado invitar a expertos a seminarios de discusión, preguntar sobre lo que consideran relevante en la disciplina pero jamás alguien que no es parte del proceso o que trabará en impulsarlo, debe responder a las preguntas. Esto no solo causará poco compromiso, sino que además no permite la creación de una perspectiva común respecto a la disciplina y el perfil profesional.

3. Identificar un caso exitoso y asumirlo como nuestro. De las tres opciones esta constituye el peor de los escenarios ya que omite en absoluto el análisis requerido para el cambio curricular y no permite tener una visión clara de qué desarrollaremos en nuestros estudiantes y porqué estamos haciendo tal o cual actividades. Se derivará en acciones que nadie comprende por qué se realizan y para qué son importantes.

En el caso de la EAP, algunas de las actividades realizadas para definir el perfil profesional y el objeto de estudio de la carrera son:

a. Consulta en talleres con docentes, estudiantes y egresados sobre las características de los profesionales y su futuro.

b. Reuniones de la Comisión de Plan de Estudios para diseñar las propuestas y sus justificaciones teóricas y prácticas.

c. Realización de actividades de consulta a profesores de otras disciplinas respecto a la administración pública. Estas actividades, incluso, se realizaron con estudiantes de la carrera quienes planteaban sus propias dudas en las entrevistas, y brindaban perspectivas que, desde el espacio docente, no percibimos como relevantes.

\footnotetext{
${ }^{6}$ Las tecnologías de información permiten una gran serie de posibilidades de interacción, por ejemplo foros, grupos de trabajo virtuales, o también semipresenciales con asignaciones de preguntas y manejo por correo con alguna persona que sistematice las percepciones del conjunto y las convierta en información relevante para la toma de decisiones.
} 
d. Realización de foros virtuales y presenciales relacionados con el tema. Esta actividad fue importante pero eran mucho más concurridas las presenciales. ${ }^{7}$

e. Validación de las propuestas con la comunidad académica a través de medios virtuales y presentaciones en la Asamblea de Escuela. ${ }^{8}$

La Escuela definió como parte de su responsabilidad social, ética y disciplinaria ser congruente con los objetivos de la Declaración Mundial sobre la Educación Superior en el siglo XXI, los cuales se resumen de la siguiente forma:

a. Tener la misión de educar, formar y realizar investigaciones.

b. Desarrollar una función ética, autónoma, responsable y prospectiva en la sociedad mundial.

c. Promover la igualdad de acceso y de oportunidades, tanto en el ámbito educativo como en la formación ética profesional.

d. Promover el saber mediante la investigación.

e. Mantener la pertinencia de la enseñanza de la administración pública, mediante planteamientos interdisciplinarios y transdisciplinarios de formación.

f. Reforzar la cooperación con el mundo del trabajo y el análisis y la previsión de necesidades de la sociedad.

g. La búsqueda constante de la integralidad para reforzar la igualdad de oportunidades (diversidad).

h. Implementar métodos educativos innovadores, como el pensamiento crítico, el pensamiento complejo y la creatividad.

i. Reforzar la idea de que el personal docente y el estudiantado son los protagonistas de la educación superior, y en el caso de la Escuela de Administración Pública bajo un enfoque pedagógico holístico que busque la construcción del conocimiento.

Como resultado de la reflexión anteriormente descrita, la Escuela redactó la siguiente definición del objeto de estudio. La Administración Pública es:

\footnotetext{
${ }^{7}$ De alguna manera esto demostró la brecha digital existente, puesto que las actividades presenciales daban mejores frutos que las virtuales. En este sentido se detecto que existían brechas tanto en el uso de las TIC así como de intermediación de procesos de reflexión y aprendizaje a través del uso de estas tecnologías.

${ }^{8}$ La Asamblea de Escuela constituye el órgano máximo de decisión de la Unidad Académica o Escuela. Se compone de profesores y profesoras que han ingresado a un Régimen Académico especial y cuentan con un nombramiento permanente en dicha Escuela.
} 
El estudio de lo público, que surge de la convergencia en la dinámica del Estado, el Mercado y la Sociedad Civil donde los actores (públicos y privados, nacionales e internacionales) construyen el bienestar general a partir de sus intereses particulares en una sociedad democrática.

Interesa el estudio de la acción pública resultante de la capacidad organizativa de los funcionarios, agencias y operadores públicos, de los agentes económicos y de la ciudadanía, que en un contexto histórico y una realidad social determinada, son capaces de construir políticas, programas y proyectos que garanticen el desarrollo humano sustentable; en el ámbito local, regional, nacional, interméstico e internacional y en un contexto globalizado. (Escuela de Administración Pública, 2011, p. 12)

El objeto de estudio que se define para el proyecto curricular es consecuente con la necesidad de comprender la acción pública desde un enfoque complejo, que responde a una sociedad dinámica, cambiante y globalizada. En términos de Morín (2009), se cambia hacia el pensamiento de una instancia planetaria, para nuestra disciplina esto supone que la acción pública ya no es exclusiva de las acciones locales, sino que el Estado se reconfigura en una nueva dinámica con el mercado y la sociedad civil más internacionalizada.

Una vez que este proceso de reflexión haya madurado, es decir, cuando existan más acuerdos que desacuerdos entre el objeto de estudio y el perfil profesional, y la comunidad académica se encuentre dispuesta y crea necesario que hay que generar un cambio, es el momento de iniciar el proceso por competencias. ${ }^{9}$

No se debe dejar de lado que el cambio de enfoque hacia competencias, organizacionalmente, se comprende como un cambio estructural y funcional que impacta directamente (y debe hacerlo) en la cultura organizacional. Esto, por cuanto el enfoque constructivista y de complejidad en los que descansan las competencias exige un cambio conceptual y metodológico respecto al paradigma tradicional educativo, y un sistema de

\footnotetext{
${ }^{9}$ El consenso unánime en nuestra experiencia nunca existió, tanto en el objeto de estudio como en el perfil profesional siempre hubo posiciones diversas, que estaban relacionadas principalmente con visiones de mundo, visiones disciplinarias y temores al cambio. Lo fundamental es buscar un equipo de impulso, crear espacios de diálogo y convencer a las personas que no se trata de pensar igual, sino de aportar al conocimiento y al desarrollo de competencias profesionales con un sentido en la sociedad.
} 
intercambio docente mucho más activo, por lo que la comunidad debe estar siendo preparada e integrada constantemente en el proceso de cambio.

\section{5 ¿Cómo se diseña el perfil profesional?}

Una vez planteado el objeto de estudio de la Escuela, que es la visualización disciplinaria de nuestro aporte a la sociedad, se procede a diseñar un perfil de profesional que responda a ese objeto, a través de competencias específicas y generales. Se retomaron las preguntas formuladas anteriormente y, con base en el objeto de estudio, se procedió a redactar el perfil de salida del y la profesional en administración pública:

Es un ser humano capaz de comprender en su accionar el contexto histórico y la realidad social que lo determina para actuar en ella; con una visión de mundo que le permita desenvolverse de forma creativa, innovadora, ética, individual y colectivamente.

Como profesional tiene la capacidad de liderar y gestionar organizaciones que desarrollan acciones de interés público.

Es capaz de construir políticas, estrategias, programas y proyectos, que garanticen el desarrollo local, regional, nacional e internacional, en un contexto globalizado. (Escuela de Administración Pública, 2011, p. 15)

El proceso para dicha redacción fue el siguiente:

1. En una sesión posterior a la de definición del objeto de estudio se volvieron a presentar las siguientes preguntas:

- ¿Cómo reconocer y traducir las necesidades de la sociedad civil desde sus espacios territoriales (local, regional y nacional, interméstico e internacional) hasta los espacios político-decisorios de manera eficiente?.

- ¿Cómo se analiza la situación económica, social, política, ambiental, normativa y cultural de un país y sus relaciones?.

- ¿Cuál es la manera más eficiente y eficaz de utilizar los recursos públicos para responder al interés común y al mejoramiento de la calidad de vida de la sociedad civil en contextos dinámicos?.

- ¿Cómo se identifican y evalúan las acciones y políticas públicas, y cómo se miden sus consecuencias?. 
- ¿Quién produce bienes y servicios considerados valiosos por los ciudadanos?. Y finalmente,

- ¿Qué es y cómo se logra el bienestar común?.

2. Se pidió luego a los docentes que redactaran características, valores, acciones y saberes relacionados con el objeto y que respondieran a las preguntas.

3. Cada miembro iba explicando qué aspectos eran relevantes y brindaban una redacción del perfil.

4. Otro compañero mientras tanto unía las ideas y determinaba puntos congruentes e incongruentes.

5. El compañero que redactaba el perfil realizó algunas preguntas para aclarar posiciones que parecían estar encontradas, conceptos diversos o aportaba aspectos que no se habían comentado.

6. Se propuso un primer diseño de perfil profesional que se fue discutiendo en plenaria hasta llegar a la versión final.

7. Posteriormente, en una sesión de trabajo con la comunidad de docentes, se presentó el objeto de estudio y el perfil de salida. En esa oportunidad se pudo mejorar con aportes de la comunidad total de compañeros.

8. Finalmente, ambos, el objeto y el perfil de salida se consolidaron y siguieron apareciendo en la mayoría de los documentos de la Escuela con el propósito de que las personas lo interioricen.

Posteriormente a la definición de objeto y perfil, se procedió a trabajar de lleno en las competencias.

\section{3. ¿Qué es una competencia?}

Actualmente, existe una gran cantidad de bibliografía relacionada con las competencias. No obstante, es importante realizar dos apreciaciones que convergen: la referente a competencias laborales y la relacionada con competencias educativas. ${ }^{10} \mathrm{La}$ primera corriente aplicada como enfoque teórico al plan curricular es la corriente de la Organización Internacional del Trabajo (OIT) cuyo espectro es amplio. De la OIT se pueden destacar las siguientes definiciones:

\footnotetext{
10 Esta diferenciación se realiza con base en la revisión bibliográfica que ha permito comprender que las competencias desde el enfoque laboral son mas exigentes en las evidencias de saber hacer; por su parte las educativas ponen especial detalle en los saberes relacionados con el ser y el conocer.
} 
- Capacidad integral que tiene una persona para desempeñarse eficazmente en situaciones específicas de trabajo (Agudelo, 1998).

- Un conjunto de propiedades en permanente modificación que deben ser sometidas a la prueba de la resolución de problemas concretos en situaciones de trabajo que entrañan ciertos márgenes de incertidumbre y complejidad técnica [...] no provienen de la aplicación de un currículum [...] sino de un ejercicio de aplicación de conocimientos en circunstancias críticas (Gallart y Jacinto, 1997).

- Una construcción, a partir de una combinación de recursos propios (conocimientos, saber hacer, cualidades o aptitudes), y recursos del ambiente (relaciones, documentos, informaciones y otros) que son movilizados para lograr un desempeño (Le Boterf, 1998).

- Aporta una interesante diferenciación entre los conceptos de calificación y competencia. Mientras por calificación se entiende el conjunto de conocimientos y habilidades que los individuos adquieren durante los procesos de socialización y formación, la competencia se refiere únicamente a ciertos aspectos del acervo de conocimientos y habilidades: los necesarios para llegar a ciertos resultados exigidos en una circunstancia determinada. La capacidad real para lograr un objetivo o resultado en un contexto dado (Mertens, 1996).

Estas definiciones se dirigen al espacio de trabajo y ponen énfasis en las capacidades de los trabajadores para ser competentes al realizar labores relacionadas con el puesto.

Por su parte, la corriente educativa tiene un enfoque hacia la incumbencia del profesional en su profesión. También se preocupa por la necesidad de que el y la discente y futuro profesional aborde problemas de su disciplina de una manera integral. Son diversas las definiciones también sobre competencias relacionadas con la planificación curricular, en el proceso se utilizaron como referencia las siguientes:

- Competencia es una actuación integral para analizar y resolver problemas del contexto (Tobón, 2009).

- Por competencia es necesario entender un complejo que implica y abarca, en cada caso, al menos cuatro componentes: información, conocimiento (en cuanto apropiación, procesamiento y aplicación de la información), habilidad y actitud o valor (Valle Flores, 2000). 
Una competencia aplicable a la disciplina de la administración pública es la capacidad de incumbencia que tiene un profesional para resolver un requerimiento de naturaleza pública, esto implica no solamente que debe saber resolverla, sino que le caracteriza la disposición permanente por hacerlo.

\section{1 ¿Cómo se redacta una competencia para la administración pública?}

La redacción de las competencias se presenta de diversas formas y, de manera general. Se puede decir que depende de la necesidad de la profesión para hacerse entender y del perfil profesional que se defina. En el caso de la Escuela de Administración Pública se siguieron las características de Tobón; primero, se definen los saberes y luego se redactan las competencias. Este perfil de salida se basa en las recomendaciones de Jaques Delors, respecto a construir el perfil con base en los cuatro pilares: aprender a conocer, aprender a hacer, aprender a vivir y aprender a ser (Delors, 1999) En el Diagrama 3 se muestra la construcción de los saberes establecidos por Delors que es parte fundamental de la propuesta de la carrera de administración pública.

\section{Diagrama 3}

Saber conocer, saber hacer y saber ser

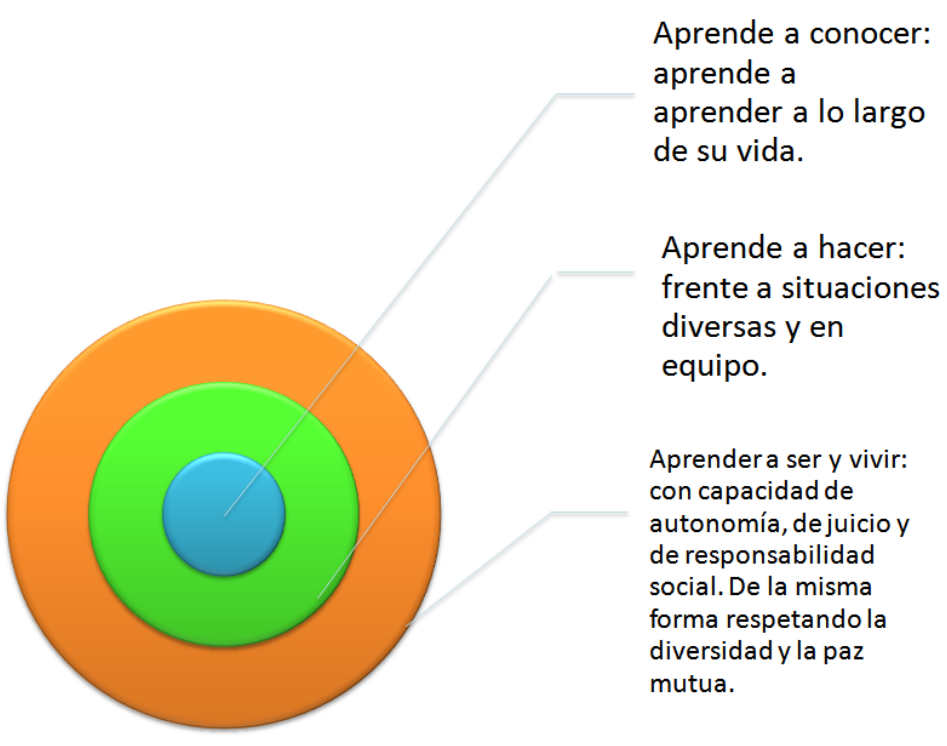

Fuente: Elaboración propia con base en Delors, 1999. 
Con base en el perfil profesional, el pensamiento complejo y el enfoque basado en competencias se delinearon el saber ser, el saber hacer y el saber conocer que caracterizan a el y a la profesional. Para efectos pedagógicos se ha decidido consolidar el saber ser y el saber vivir. La tabla 1 resume los principales acuerdos respecto a los tres saberes establecidos por Delors, 1999 y aplicados al contexto de la administración pública.

\section{Tabla 1}

Saber ser, saber hacer y saber conocer aplicado al Perfil profesional Proyecto Curricular de Administración Pública

\begin{tabular}{|c|c|c|}
\hline $\begin{array}{l}\text { SABER } \\
\text { SER }\end{array}$ & $\begin{array}{l}\text { Construye } \\
\text { un } \\
\text { proyecto } \\
\text { ético de } \\
\text { vida }\end{array}$ & $\begin{array}{l}\text { El y la profesional en administración pública reconoce la importancia de } \\
\text { tener un proyecto de vida que le permita comprender su papel en la } \\
\text { sociedad. Dirige su carrera hacia temas que le son interesantes, } \\
\text { relevantes para su vida y que promueven su curiosidad } \\
\text { permanentemente. } \\
\text { En el contexto social, reconoce el valor de la organización ciudadana } \\
\text { para el logro del bienestar común; y es un y una agente de cambio capaz } \\
\text { de comprender necesidades, relaciones y soluciones. } \\
\text { Se espera que el y la profesional desarrolle durante su educación } \\
\text { superior las capacidades para establecer sus metas personales y las de } \\
\text { su comunidad; todo esto en un marco ético de acción. } \\
\text { Como ciudadano y profesional reconoce sus capacidades y desafíos, que } \\
\text { le permiten evolucionar permanentemente a través de valores como la } \\
\text { crítica constructiva, la honestidad, el valor del servicio a la comunidad, la } \\
\text { perseverancia y la paz. }\end{array}$ \\
\hline & $\begin{array}{l}\text { Conoce sus } \\
\text { habilidades } \\
\text { y destrezas } \\
\text { para el } \\
\text { desempeño } \\
\text { profesional }\end{array}$ & $\begin{array}{l}\text { Escoge la mejor forma de usar los recursos públicos, con base en criterios } \\
\text { éticos y técnicos. } \\
\text { Comprende la organización social con fines públicos; la conoce, analiza y } \\
\text { evalúa de manera efectiva. } \\
\text { Gestiona e implementa las técnicas y herramientas necesarias que optimicen } \\
\text { el uso de los recursos públicos en las organizaciones; es decir, aplica y crea } \\
\text { efectivamente tecnología administrativa. } \\
\text { Comprende y desarrolla de manera efectiva el proceso de políticas públicas. } \\
\text { Reconoce el valor del trabajo interdisciplinario en la resolución de problemas } \\
\text { públicos, lo desarrolla a través del trabajo en equipo. } \\
\text { Tiene la capacidad de aplicar los principios éticos y de calidad a las actividades } \\
\text { relacionadas con la función pública, en busca del bienestar general. } \\
\text { Diseña y aplica la estrategia financiera que permite el logro de objetivos y la } \\
\text { prestación de servicios. }\end{array}$ \\
\hline $\begin{array}{l}\text { SABER } \\
\text { CONOCER }\end{array}$ & $\begin{array}{l}\text { Desarrolla } \\
\text { su } \\
\text { capacidad } \\
\text { cognoscitiva }\end{array}$ & $\begin{array}{l}\text { Es un y una profesional integral, que comprende la dinámica global de la } \\
\text { sociedad a través de las herramientas desarrolladas en su carrera. } \\
\text { Es capaz de comprender las características de la sociedad en que se } \\
\text { desenvuelve, los cambios en ésta y el sistema de interrelaciones de poder } \\
\text { social y organizacional; todo esto con el fin de tomar las mejores decisiones de } \\
\text { incidencia pública. } \\
\text { Desarrolla habilidades de intercambio con quienes le rodean, e incide } \\
\text { positivamente en los intercambios sociales de la función pública. } \\
\text { En síntesis analiza, el entorno y el contexto, sistematiza, aprende y crea } \\
\text { conocimiento significativo para si, su organización y la disciplina de la } \\
\text { administración pública. }\end{array}$ \\
\hline
\end{tabular}

Fuente: Elaboración de la Comisión de Plan de Estudios con base en trabajo colaborativo docente y basado en formato de resolución VD-R-8411-2010. 
De acuerdo con Tobón, 2009 la redacción de cada competencia debe cumplir con cuatro elementos: verbo, objeto conceptual, finalidad de la competencia y condición de calidad. En el Diagrama 4 se ejemplifica la redacción de la competencia y sus insumos con base en las recomendaciones del autor.

\section{Diagrama 4}

\section{Redacción de las competencias, sus componentes y fuentes de información}

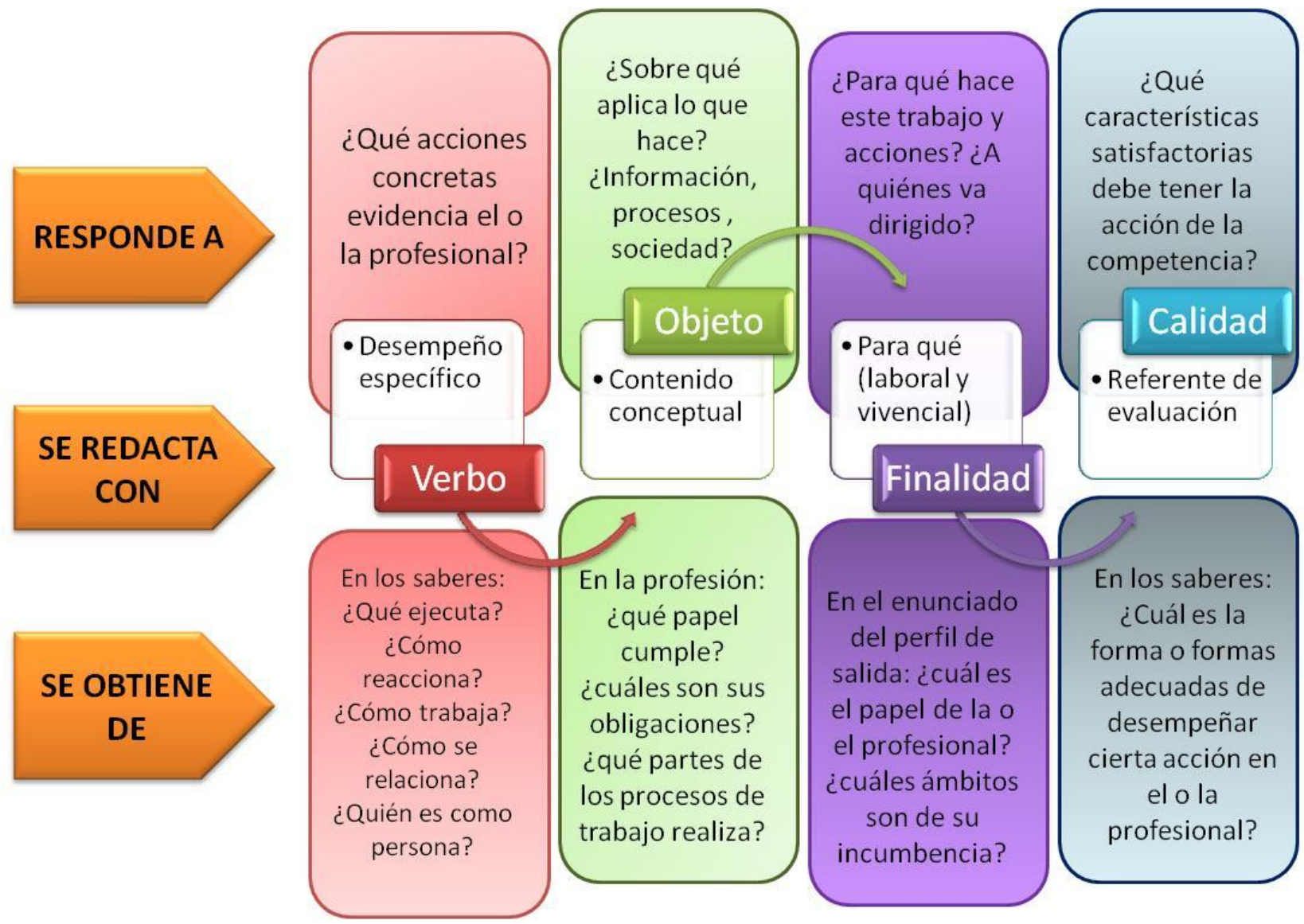

Fuente: Elaboración propia con base en elementos de Tobón, 2009.

Tomando como base la conexión de ideas del Diagrama 4, la Comisión procedió a redactar las competencias. En este caso, son de dos tipos: generales y específicas. Las competencias generales se refieren al grupo de capacidades que cualquier profesional debe cumplir; por ejemplo, trabajo en equipos, comunicación, adaptación al cambio. Una buena fuente de análisis de competencias generales es la Organización Internacional del Trabajo que cuenta con un diccionario de competencias que puede ser útil de revisar. 
Sin embargo, solo después del análisis del perfil se definen cuales son las competencias relevantes, esto, por cuanto algunas competencias consideradas generales deberían tener una variación para adaptarla a la profesión y en ese momento se convertirían en una competencia específica. Por consiguiente, las competencias específicas van enfocadas a aspectos relacionados directamente con la profesión y sus particularidades.

Muestra de esta diferencia es la competencia de comunicación. Esta puede ayudar de la misma forma a un profesional de contabilidad o administración, quienes trabajan con personas con fines organizativos. Por su parte, en el área de psicología o medicina psiquiátrica, la competencia no sería exactamente la misma, ya que está dirigida a la atención de un paciente específico y este, a su vez, responde a necesidades del medio; es decir, son perfiles profesionales diferentes. Incluso para el profesional de psicología se pueden establecer dos competencias relacionadas con la comunicación, una para el trabajo con compañeros de trabajo en el logro de objetivos organizacionales (general) y otra relacionada con el tipo de atención brindada a los pacientes (específica).

Por esta razon, esde suma importancia el análisis que se explicó anteriormente puesto que hay muchas competencias definidas que resultan interesantes, pero solo funcionan las que genuinamente parten del perfil profesional que se quiere obtener. Otro ejemplo es que la competencia de comunicación de un vendedor tiene fines de persuación, mientras que para otro profesional no necesariamente le interese convencer, sino simplemente entregar un mensaje de forma clara.

En el caso de EAP, el proceso de confección de competencias se realizó en equipos docentes y se puede resumir en los siguientes pasos:

1. Se convocó a una sesión de docentes para trabajar el diseño de las competencias generales y específicas.

2. Los profesores y las profesoras se dividieron en dos grandes grupos: competencias generales y competencias específicas, estos a la vez se dividieron en dos grupos para garantizar la participación de todos. Para esto, se construyeron una serie de instrumentos basados en el perfil donde se guiaba a los equipos hasta llegar a la redacción de las competencias.

3. En plenaria, cada equipo fue exponiendo sus competencias y explicando la importancia y razones por las que lo proponían.

4. Uno de los docentes, especialista en la técnica de METAPLAN, aportó al grupo sistematizando las propuestas. 
5. Rápidamente comenzaron los equipos de cada tipo de competencia a tener puntos de encuentro, los cuales al final se sintetizaron en las competencias. En ese momento se definieron aproximadamente unas 35 competencias.

6. La Comisión de Plan de estudios procedió a afinar, en la siguiente sesión, el producto y presentarlo nuevamente en la siguiente sesión a los compañeros y a las compañeras.

7. En el trabajo de la comisión se revisó cada una de las competencias con tres fines: que estuvieran bien redactadas, que se relacionaran con el perfil de salida y que no se repitieran. Al finalizar este proceso quedaron diez competencias generales y diez más específicas.

La Comisión consideró que el manejo de veinte competencias era demasiado y que se debía hacer un último intento por ser más sintéticos y disminuir en cantidad las competencias. En la siguiente sesión con todos los profesores, se validaron las competencias y además se pidió a los compañeros que priorizaran nuevamente, buscando tendencias o evidencias de paralelismos. La sorpresa es que ninguna de las competencias obtuvo tendencias tan importantes como para descartar otras, es decir la priorización no funcionó.

Al consultarse a diversos expertos respecto a la cantidad de competencias, las principales respuestas se enfocaron en la importancia de que fuera un producto propio.

La decisión tomada fue que era necesario mantener las competencias y en cada evaluación anual revisarlas junto a sus niveles, de tal forma que el aprendizaje en el proceso de implementación permitiera validarlas.

\section{2 ¿Qué son los niveles de las competencias y cómo se definen?}

Para que un profesional desarrolle una competencia debe seguir un proceso que evidencia desempeños de avance y que, por lo tanto, permita definir secuencias durante el desarrollo de esta. Por ejemplo, en el aprendizaje para caminar, el cual aplica para niños o adultos que han tenido que aprender y reaprender, el alcanzar la competencia de caminar o correr lleva secuencias que podrían ser aprender a desplazarse (gatear), levantarse y mantenerse de pie por un rato, caminar sosteniéndose de alguna estructura y finalmente, caminar. Así, en los niños se requiere que se sigan de forma general todos los pasos pero el tiempo tomado es relativo, ya que depende de qué tan sencillo o traumático sea el proceso, 
también de la disposición del espacio y de las condiciones de apoyo de sus padres y hermanos.

En el caso de las competencias profesionales, la secuencia se define en logros de avance que pueden ser vistos en la forma de trabajar (técnicas) o de actuar (personales) del individuo. De este modo, el resultado de las competencias se presentó de acuerdo con el formato de la Tabla 2.

\section{Tabla 2}

\section{Competencia de trabajo en Equipo}

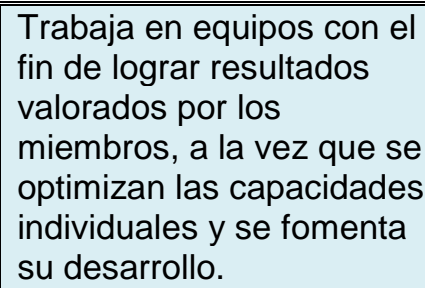

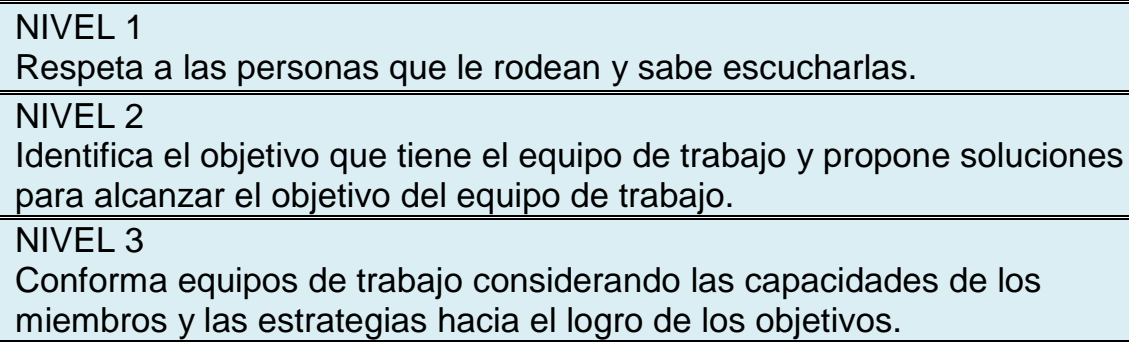

Fuente: Escuela de Administración Pública, 2011

La cantidad de niveles de la competencia se define por el equipo de personas que la diseñan. Para definir su redacción es importante considerar cuáles actitudes y acciones debe evidenciar o demostrar la persona cuando ésta ha alcanzado el nivel más alto. Por ejemplo en la competencia de trabajo en equipo el enfoque es organizacional y de logro de resultados, los niveles inician con crear un ambiente de respeto (toma papel de seguidor 0 miembro activo), posteriormente, hacia promover que como equipo se llegue a resultados (sin necesidad de ser líder, trabajo colaborativo) y finalmente, constituirse como un agente que construye equipos de trabajo (puede ser en papel de liderazgo).

La competencia y la definición las evidencias de logro para cada nivel va a depender del perfil de salida. Es importante reforzar el hecho de que para el caso académico este perfil está contenido en el objeto de estudio disciplinario.

Los pasos a seguir para definir los niveles son:

a. Analizar la competencia y preguntarse ¿Es posible que una persona alcance rápidamente esta competencia? ¿Qué se requiere que vaya evidenciando mientras transita en la competencia? ¿Debe aprender algo para alcanzar la competencia? ¿qué? También puede preguntarse ¿Qué conocimientos aplica una persona que domina el tema que analizamos? ¿Estas manifestaciones son dependientes 0 
interdependientes unas de otras? ¿Es posible construir un proceso general de desarrollo de la competencia?

b. Cuando se encuentran hechos para todas las competencias se divulgan a la comunidad docente y se validan.

c. Cuanto más sencillo sea visibilizar con acciones definidas y concretas lo que esa persona hace o evidencia, el nivel estará mejor definido.

\subsection{1 ¿Cómo integro competencias y contenidos?}

El definir las competencias y sus niveles es solo el principio de este viaje pero es un gran insumo. No solamente es información, haber llegado a esta parte del camino implica un nivel de reflexión que no es propio de la planeación tradicional. Probablemente usted y sus compañeros y compañeras estén más claros respecto a la disciplina, al perfil que desean, y también tienen mayor consciencia de lo que no tienen claro.

Una de las ventajas de los procesos de reflexión por competencias es la capacidad de construir y aprender construyendo. En este momento, usted sabe en qué aspectos la mayoría de los miembros están claros, satisfechos, preocupados e interesados por mejorar. Esto permite que la siguiente etapa, la de planificación propiamente, sea un verdadero trabajo en equipo.

Normalmente, la lógica de los planes tradicionales nos orienta a definir áreas y luego temas que deben saber los y las profesionales para desempeñarse en el ámbito laboral.

El desarrollo de la malla desde los temas, conlleva el riesgo que normalmente se presenta, y es que no exista concatenación entre los temas. Por consiguiente, los estudiantes llevan diversas materias que indican conocimientos relevantes para la carrera en general, pero en muchas ocasiones no se tiene claridad de cómo estas materias interactúan. En el mejor de los casos existen coordinaciones de áreas y niveles, lo que ordena el seguimiento de los temas pero puede dejar de lado las destrezas y habilidades. En otras ocasiones, las materias no son ofrecidas directamente por la Escuela, sino que pertenecen a otra Unidad Académica que ofrece un conjunto de conocimientos, los cuales en muchas ocasiones no son relevantes para los procesos de aprendizaje de nuestro perfil profesional.

En el caso del plan 1999-2009 de la EAP tenían materias de matemáticas enfocadas en administración general, determinación de costos, precios, porcentajes. Sin embargo, por la ubicación de la materia en el principio de la carrera se perdía el efecto, puesto que hasta dos años después los estudiantes llevaban materias relacionadas con flujos de efectivo, 
proyectos y cálculos. Muchos de los estudiantes indicaron que las materias de matemática les resultaban totalmente inútiles ya que cuando llegaban las materias relacionadas, ellos había perdido práctica.

Otro aspecto a considerar es la existencia de materias de enfoque teórico, que normalmente se brindan con un enfoque magistral. Durante la discusión hacia el cambio por competencias, algunos profesores defendían el hecho de que los estudiantes deben dominar la teoría para comprender la práctica, lo cual es totalmente cierto. No obstante, lo que es falso es que deban existir cursos totalmente teóricos que no le permitan al estudiante comprender por qué esta aprendiendo eso y para qué le servirá en su vida profesional. Si se trata de Historia por ejemplo, debe tener la capacidad de comprender por qué se estudia la historia, qué aprenderá de ella, qué métodos se siguieron para analizar un momento determinado y cómo se llegó a conclusiones específicas; es decir, hay que enseñar a hacer. Es importante recordar que las competencias se enfocan en tres aspectos: saber hacer, saber ser y saber conocer, y esto debe estar presente en el diseño de la malla final.

Por lo anterior, es necesario romper el paradigma de áreas y temas que se convierten en materias para el diseño curricular y enfocarse en la interacción del profesional en su entorno. Existen diversas formas de realizar la relación entre contenidos y competencias, sin embargo, una de las más utilizadas, tanto para competencias técnicas como profesionales se basa en los procesos de trabajo en los que se define que el o la profesional participará.

\section{Conclusiones}

Con base en la sistematización de esta experiencia es importante rescatar los siguientes elementos:

a. Las competencias son una forma o enfoque que resulta útil para construir el currículo académico y las estrategias curriculares universitarias. En el caso de la carrera de administración pública, el proceso ha demostrado capacidad instalada para desarrollarlo y liderazgo compartido para implementarlo desde el año 2010.

b. En este artículo se comparte únicamente el proceso de definición de competencias puesto que en la bibliografía se subestima su relevancia. En muchos de los casos se indica la relevancia de realizar un plan por competencias y se va directo a la malla curricular, lo cual constituye un error el pasar por alto esta dinámica.

c. La clara definición del objeto de estudio de la disciplina y la construcción consensuada del perfil profesional serán determinantes en la confección curricular y su posterior 
implementación. Sin estos dos elementos claros en la comunidad académica la propuesta sufrirá los vaivenes de procesos de redefinición de la disciplina y de las demandas de la sociedad.

d. Las experiencias curriculares que descansen en una sola persona, posiblemente, llegarán a fracasar puesto que las competencias implican un esfuerzo colectivo de trabajo y de pensamiento creativo.

e. La sistematización del proceso es importante y utilizar sistemas de información desde el inicio es una excelente decisión. De la misma forma, encontrar medios de intercambio basados en tecnologías de la información (chats, foros, correos, wikis) es de gran ayuda para ahorrar costos.

f. Finalmente, este es un proceso de cambio organizacional y debe ser abordado como tal.

\section{Referencias}

Agudelo, Santiago. (1998). Certificación de competencias laborales, Aplicación en Gastronomía. Montevideo: CINTERFOR/ OIT.

CONARE. (17 de abril de 2013). Consejo Nacional de Rectores. Recuperado de http://www.conare.ac.cr/

Delors, Jaques. (1999). La educación encierra un tesoro: Informe a la UNESCO de la Comisión Internacional sobre la Educación para el Siglo XXI. Francia:Ediciones UNESCO.

Escudero, Juan Manuel. (2009). Diseño, desarrollo einnovación del currículum. Madrid: Sintesis Educación.

Escuela de Administración Pública. (2011). Cambio Plan Curricular. Escuela de Administración Pública, Universidad de Costa Rica, documento de trabajo.

Gallart, María Antonia y Jacinto, Claudia. (1997). Competencias laborales: tema clave en la articulación educación trabajo. En Cuestiones actuales de la formación. Montevideo: Cinterfor/ OIT.

Le Boterf, Guy. (1998). La ingeniería de las competencias. París: D’organisation.

Livinstong, Joan Sterling. (1971). El Mito del Gerente Instruido. Harvard Bussines Review, 79.

Mertens, Leonard. (1996). Competencia Laboral: sistemas, surgimiento y modelos. Montevideo: CINTERFOR/ OIT. 
Morín, Edgar. (2009). Complejidad y Sujeto Humano. "El sujeto humano, la acción y la ética". Recuperado de http://www.edgarmorin.org/images/publicaciones/etica.pdf

Ortegón, Edgar, Pacheco, Juan Francisco y Prieto, Adriana. (2005). Metodología del marco lógico para la planificación, el seguimiento y la evaluación de proyectos y programas. Santiago, Chile: ILPES CEPAL.

Tobón, Sergio. (2009). Manual sisntético de gestión curricular por competencias. Bogota: Cife.

Valle Flores, María de los Angeles. (2000). Formación en competencias y certificación profesional. Mexico: UNAM. 